\title{
Lateral instability in normal viscous fingers
}

\author{
R. Ledesma-Aguilar, M. Quevedo-Reyes, and E. Corvera Poiré \\ Departamento de Física y Química Teórica, Facultad de Química, UNAM Ciudad Universitaria, México Distrito Federal 04510, Mexico
}

\author{
A. Hernández-Machado
}

Departament ECM, Facultat de Física, Universitat de Barcelona, Diagonal 647, E-08028 Barcelona, Spain

(Received 28 April 2004; revised manuscript received 23 August 2004; published 27 January 2005)

\begin{abstract}
We study a low-amplitude, long-wavelength lateral instability of the Saffman-Taylor finger by means of a phase-field model. We observe such an instability in two situations in which small dynamic perturbations are overimposed to a constant pressure drop. We first study the case in which the perturbation consists of a single oscillatory mode and then a case in which the perturbation consists of temporal noise. In both cases the instability undergoes a process of selection.
\end{abstract}

DOI: 10.1103/PhysRevE.71.016312

PACS number(s): 47.54.+r, 05.10.- a, 47.55.Mh

\section{INTRODUCTION}

Branching is an ubiquitous process in nature [1-4]. From plants, rivers, blood vessels, and bacterial colonies to dendritic growth, the universality of branching has been put forward. In crystal growth, when an undercooled melt solidifies, the solid front has a parabolic shape; lateral protrusions, called sidebranches, form on the main structure and grow in amplitude as they are advected away from the parabolic tip. This phenomenon has been widely investigated in solidification. It has been proposed, both experimentally $[5,6]$ and theoretically [7-9] that sidebranching results from selective amplification of natural noise.

Natural noise, that is, noise unintentionally present in the system, is not enough to create sidebranching in normal Saffman-Taylor fingers. Normal Saffman-Taylor fingers appear when an inviscid fluid displaces a viscous fluid in a Hele-Shaw cell, a pair of glass plates parallel to each other that form an almost two-dimensional channel in which the flow takes place. In the absence of perturbations, SaffmanTaylor fingers are always greater than half of the channel width. We refer to these steady-state fingers as normal Saffman-Taylor fingers, to be able to differentiate them from anomalous fingers, whose width is less than half of the channel width and that are observed when anisotropy is imposed on the system. In anomalous Saffman-Taylor fingers, dendrites have been observed using localized disturbances, such as a bubble placed at the finger tip or a thread placed along the channel $[10,11]$. In both dendritic crystals and anomalous fingers, it has been shown that periodic forcing induces periodic sidebranches [12-14]. Experimentally, sidebranches have been observed in viscous fingers for miscible fluids in a radial cell when anisotropy is imposed on the system by engraving a grid on one of the plates $[15,16]$. For immiscible fluids, sidebranches are strongly suppressed by surface tension, and a low-amplitude lateral instability can be observed [17]. Theoretically, viscous fingers in the radial cell with fourfold anisotropy have been studied in Ref. [18]. In agreement with experiments, regimes for which fingers have lateral instabilities, whose amplitude is strongly suppressed by surface tension, are found. Localized nonlinear instabilities of the normal Saffman-Taylor finger have also been reported
[19]. Recently, fluctuations along the finger sides have been reported in normal fingers for low capillary numbers and very wide and long channels [20].

A wide variety of problems, which include biorheology and oil recovery, involve the dynamics of confined fluids in frequency-dependent flow regimes. Due to its relative simplicity, the Saffman-Taylor finger is an archetype of both pattern-forming systems and two-phase flow in confined systems [21]. We are, therefore, interested on the response of the Saffman-Taylor finger to a controlled frequency-dependent flow.

Here we report a lateral instability of the normal SaffmanTaylor finger induced dynamically in a controlled manner. We also propose for which frequencies and cell dimensions this instability can be more easily experimentally observed. By means of a phase-field model, we study two cases. The first one, in which an oscillatory signal is overimposed to a constant pressure gradient, leads to a strictly periodic lateral instability that undergoes a process of selection. We then study the effect that dynamic noise has on the finger shape and observe a similar lateral instability. This one, despite its nonperiodicity, undergoes a process of selection as in the first case.

\section{PHASE-FIELD MODEL AND MACROSCOPIC MODEL}

Recent studies indicate that the Saffman-Taylor problem for Newtonian fluids can be successfully studied by means of phase-field models [22-24]. The main advantage of such mesoscopic approaches is that they avoid complicated methods for tracking the interface. In particular, it has been found that a single-order-parameter equation suffices to reproduce the macroscopic equations of the Saffman-Taylor problem in the infinite-viscosity-contrast limit [24]. Moreover, numerical simulations of this model have reproduced the behavior of the fluid-fluid interface, from destabilization and mode competition to the formation of the steady state. We therefore decide to use the phase-field model of Ref. [24] with a boundary condition modified to allow for dynamic pressure drops. 


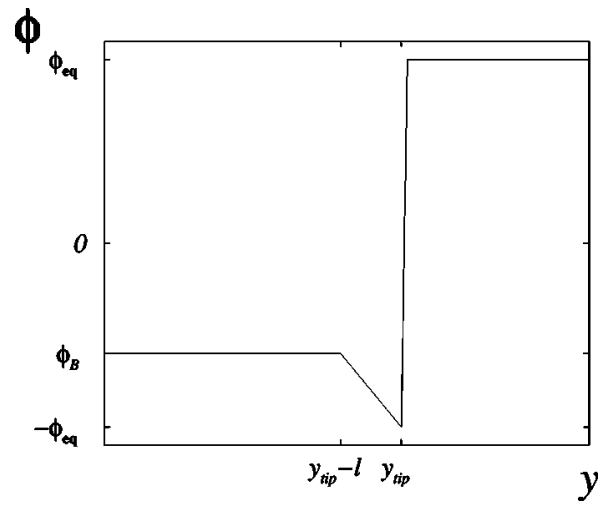

FIG. 1. Profile for the order parameter along the flow direction for the initial boundary condition. The flow takes place from the right to the left.

The mesoscopic equation to be solved for the infiniteviscosity-contrast Saffman-Taylor problem is the equation of Model B

$$
\frac{\partial \phi}{\partial t}=\nabla \cdot[M \nabla \mu(\phi)]
$$

where $\phi$ is an order parameter and $\mu(\phi)$ is a chemical potential that has the explicit form

$$
\mu(\phi)=-\phi+\phi^{3}-\epsilon^{2} \nabla^{2} \phi,
$$

where $\epsilon$ is a parameter proportional to the interface width. The parameter $M$ has a constant value in the viscous fluid and is zero in air. The following boundary condition is imposed at the bulk of the displaced fluid,

$$
\phi\left(x, y \leqslant y_{t i p}-l, t\right)=\phi_{B}(t) .
$$

Equation (3) fixes the bulk value $\phi_{B}(t)$ at an arbitrary distance $l$ from the fingertip. $y_{\text {tip }}$ corresponds to the most advanced point of the interface. This creates a ramp that represents the driving force of the system. The initial boundary condition to generate a steady-state finger is shown in Fig. 1. Despite their simplicity, these equations describe the hydrodynamic equations of the macroscopic problem in the sharp interface limit.

The macroscopic equations of the problem are Laplace's equation for the pressure $p$, i.e.,

$$
\nabla^{2} p=0
$$

which is written from Darcy's Law plus the incompressibility of fluids, and the boundary conditions at the fluid-fluid interface; that is, the continuity boundary condition and the local thermodynamic equilibrium condition, i.e.,

$$
\begin{gathered}
v_{n}=-\frac{b^{2}}{12 \eta} \nabla p \cdot \hat{\mathbf{n}}, \\
\Delta p=-\gamma \kappa,
\end{gathered}
$$

where $v_{n}$ is the normal velocity of the interface, $\eta$ is the viscosity of the viscous fluid, $b$ is the gap between the cell plates, $\hat{\mathbf{n}}$ is a vector normal to the interface, $\Delta p$ is the pres- sure drop at the interface, $\gamma$ is the surface tension, and $\kappa$ is the local curvature of the interface. $K \equiv b^{2} / 12 \eta$ is commonly known as the permeability of the system.

In order to recover the macroscopic equations from the mesoscopic equations, a matched asymptotic expansion is necessary and the following identification of parameters has to be made: $p=\phi_{e q} \mu_{1}, K=M / 2 \phi_{e q}^{2}$, and $\gamma=\phi_{e q}\left(\gamma^{\prime} / \Delta \phi\right)$, where $\mu_{1}$ is the first order in $\epsilon$ term of the chemical potential, $\phi_{0}$ is the zero order in $\epsilon$ term of the order parameter, $\Delta \phi$ $=2 \phi_{e q}, \phi_{e q}$ is the bulk value for the order parameter in equilibrium, and $\gamma^{\prime}=\int_{-\infty}^{\infty}\left(\partial \phi_{0} / \partial w\right) d w$. The variable $w$ is an inner coordinate of the interface, which is introduced in the expansion, and that is, at any point, perpendicular to it.

It is worth to note that in order to study the effect of a dynamic pressure drop on the Saffman finger shape, the pressure gradient should contain a constant term responsible for the finger formation. That is, the pressure gradient should be, at any time, negative so the fluid-fluid interface remains unstable. Moreover, the time-dependent term of the pressure gradient should be small compared to the constant term of the pressure gradient because we are interested in studying situations in which the single-finger solution exists. In our model, the above considerations are implemented by taking the boundary condition (3) for the order parameter in front of the finger as a constant plus a dynamic term of the form

$$
\phi_{B}(t)=\phi_{B 0}+\delta g(t)
$$

where $g(t)$ is a time-dependent dimensionless function that varies between -1 and 1 and will be considered in two different ways as explained below, and $\delta$ is an amplitude that is small compared to $\phi_{B 0}$. In the two cases described below, we have carried on the numerical integration of Eq. (1) subject to the proper dynamic boundary condition (7). We have used an Euler method for a discrete square lattice of size $n_{x} \times n_{y}$ with mesh size $\Delta x=1$ and time step $\Delta t=0.01 . n_{x}$ has been chosen to be $n_{x}=32$ in all cases, and $n_{y}$, which is the dimension along which the finger propagates, has been chosen in such a way that the lateral instability is well developed. It will be specified in each case.

\section{NATURAL FREQUENCIES}

Before describing the dynamic signals considered in the present paper, it is convenient to remember that there are two natural frequencies of the steady-state problem. The first characteristic frequency of the steady-state problem is the finger velocity divided by the finger width, which gives an angular frequency equal to

$$
\omega_{\text {finger }}=2 \pi \frac{U}{\lambda W}
$$

The other frequency characteristic of the steady-state problem is the one determined by the flow very far from the finger tip, that is, the flow velocity at infinity divided by the channel width, which gives an angular frequency equal to 

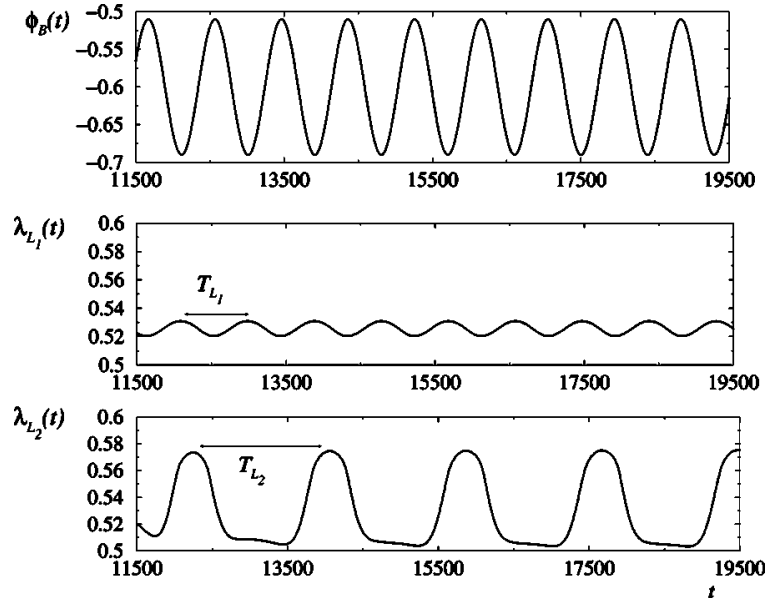

FIG. 2. Bulk value $\phi_{B}$ and finger widths measured at distances $L_{1}$ and $L_{2}$ from the fingertip plotted vs time. The mode selection process has taken place in between the second and third plots. The output signal frequencies $\omega_{L_{1}}$ and $\omega_{L_{2}}$ are determined from the periods $T_{L_{1}}$ and $T_{L_{2}}$. The incident frequency is $\omega=0.007$.

$$
\omega_{\infty}=2 \pi \frac{V_{\infty}}{W}
$$

These two frequencies are related to each other because conservation of matter implies that the finger velocity times the finger width is equal to the velocity at infinity; that, is $U \lambda$ $=V_{\infty}$.

\section{NUMERICAL RESULTS}

\section{A. Oscillatory pressure drop}

The dynamic part of the incident signal consists of a single-mode oscillatory term. That is $g(t)$ of the boundary condition (7) is

$$
g(t)=\cos (\omega t)
$$

The initial condition consists of a $\phi(x, y)$ profile that corresponds to a steady-state finger. The parameters $\phi_{B 0}$ and $\delta$ in Eq. (7) have been set equal to $\phi_{B 0}=-0.6$ and $\delta=0.09$.

As the bulk value of $\phi_{B}(t)$ oscillates, the finger responds by generating a wave on its tip. This wave is advected far from the fingertip in such a way that the sides of the finger

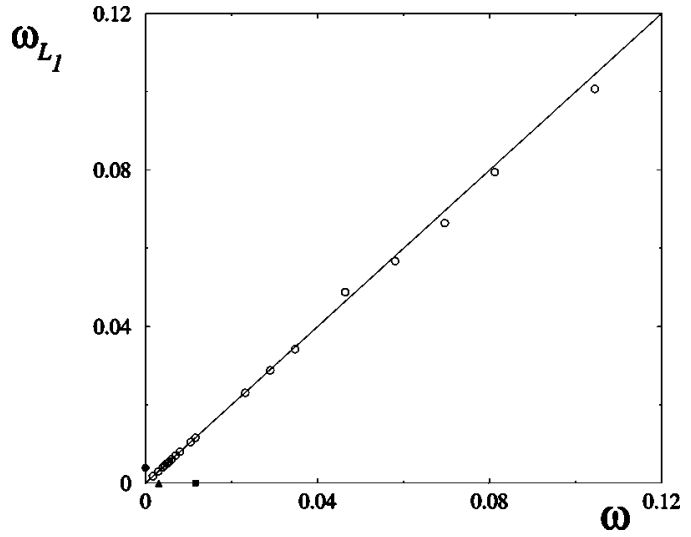

FIG. 4. Response frequency measured close to the fingertip plotted against incident frequency. For reference, the selected frequency is indicated in the coordinate axis as a circle. The natural frequencies of the flow $\omega_{\infty}$ (triangle) and $\omega_{\text {finger }}$ (square) are indicated in the abscissa axis.

are no longer flat, as in the steady-state case, but develop an instability. In order to quantify the process of growth, the finger width is measured simultaneously at two different distances from the fingertip $L_{1}$ and $L_{2}$. Due to the growth process, variations on the finger width measured at distance $L_{1}$, close to the finger tip, have a much smaller amplitude than the ones measured at distance $L_{2}$, far from the finger tip. Figure 2 illustrates the behavior of the finger width at distances $L_{1}$ and $L_{2}$ as a function of time. The incident signal on the boundary condition of the bulk order parameter has also been plotted in order to show that the incident frequency and the response frequency close to the fingertip are the same; thus the wave generated at the finger tip responds linearly to the incident signal. It can also be observed that at a distance far from the tip, the frequency of the lateral instability no longer follows the incident signal; in fact, it undergoes a dynamic process as will be discussed below. Just as with the frequency close to the fingertip, the wavelength of the lateral instability has a linear behavior; that is, it corresponds to the average finger velocity divided by the incident frequency. However, as the wave travels away from the tip toward the sides of the finger, the wavelength of the instability coarsens and reaches a value that is independent of the incident frequency. This happens for a very wide range of incident frequencies, that is, the lateral instability undergoes a mode selection process.

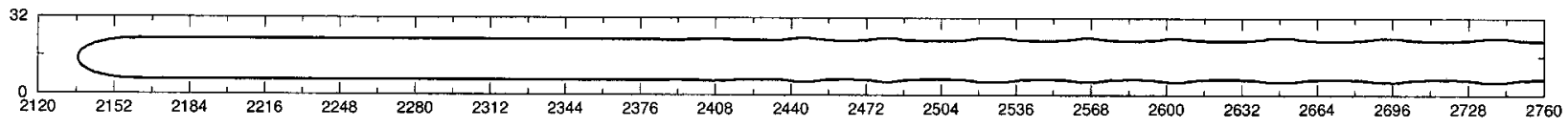

(a)
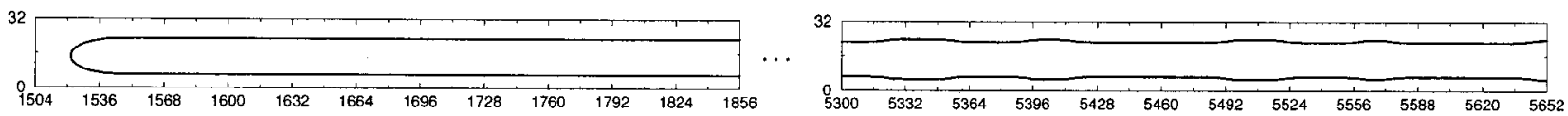

(b)

FIG. 3. Finger profiles with lateral instabilities. (a) The profile at the top is obtained when introducing an oscillatory perturbation in the pressure drop. In this case, the instability remains strictly periodic. For this simulation, $n_{y}=3200$. (b) The profile at the bottom corresponds to a pressure drop with superimposed noise in time. As a result a nonperiodic instability develops. For this simulation, $n_{y}=7000$. 


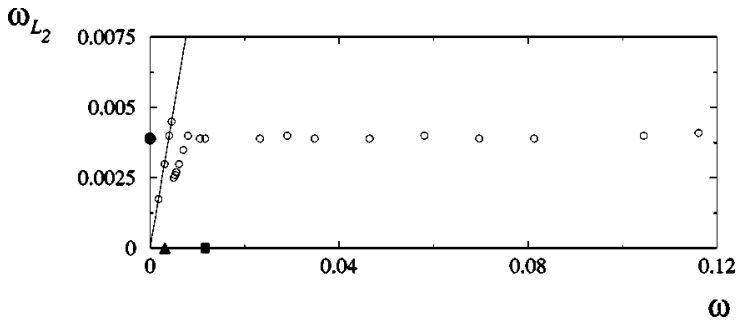

FIG. 5. Response frequency measured far from the fingertip plotted against incident frequency. For a wide range of frequencies mode selection occurs. The symbols on the axis are as in Fig. 4.

In our problem, the frequency selected for the lateral instability is roughly the characteristic frequency of the flow far from the finger tip $\omega_{\infty}$ given by Eq. (9). The amplitude of the selected mode saturates and is small compared to the finger width. Also it is a longwavelength instability as will be discussed. Therefore, the lateral instability does not have a dramatic visual effect on the finger as does the instability which causes sidebranching in solidification. However, it causes the finger to have small perturbations on its width. The instability is shown in Fig. 3(a) on a 1:1 scale in order to realize the actual size of the width variations that would be observable in an experiment.

Figure 4 shows how the frequency of the lateral instability close to the fingertip $\omega_{L_{1}}$ is the same as the incident frequency $\omega$; that is, $\omega_{L_{1}}=\omega$. This frequency is measured from the signal period $T_{L_{1}}$ with the relation $\omega_{L_{1}}=2 \pi / T_{L_{1}}$. This linear response close to the finger tip, has been observed for all of the incident frequencies studied. $L_{1}$ has been set equal to $L_{1}=40 \Delta x$.

The process of frequency selection far from the finger tip is shown in Fig. 5. The frequency of the lateral instability $\omega_{L_{2}}$ has the same value for a wide range of incident frequencies. This frequency is slightly larger than the characteristic frequency of the flow at infinity given in Eq. (9). The frequency is measured from the signal period $T_{L_{2}}$ using $\omega_{L_{2}}$ $=2 \pi / T_{L_{2}}$. The continuous line is shown for reference and is the line that would correspond to linear response. We can clearly see that there are three regimes for the response frequency. First, at very low incident frequencies, the response frequency is always equal to the incident frequency. In this region, surface tension is not enough to suppress the modes that cause a perturbation to the sides of the finger. Second,

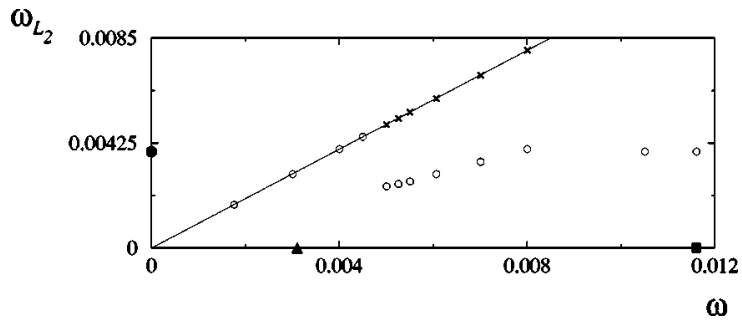

FIG. 6. Linear and transition zones of the $\omega_{L_{2}}$ vs $\omega$ curve. Circles correspond to simulation results and crosses represent the double of the observed frequency $2 \omega_{L_{2}}$, which fall on the diagonal and make evident that the first harmonic of the incident signal has grown. The symbols on the axes correspond to the same frequencies as in Fig. 4.

there is a range of frequencies for which there is a behavior between linear response and selection. In this range of transition, the response frequency corresponds in all cases to half the incident frequency. This is shown in Fig. 6. We can understand this behavior if we think that in this region, surface tension is large enough to suppress the incident frequency, but not large enough to suppress its first harmonic. Finally, we see that for incident frequencies larger that the characteristic frequency of the finger $\omega_{\text {finger }}$ given by Eq. (8), the response frequency is independent of the incident frequency; there is a mode that is selected because it grows faster than the others. The selected frequency determines the wavelength of the lateral instability.

It is important to note that the lateral instability appears, in many cases, far from the finger tip. For instance, in the region of incident frequencies where selection is observed, the amplitude of the lateral instability saturates at a distance of the order of ten times the cell width and is very small close to the finger tip. See Fig. 3(a). For the linear and the transition zones (Fig. 7), the distance from the tip at which the lateral instability is observed is much smaller as will be discussed later. The distance $L_{2}$ has been chosen in each case as to measure the instability once the amplitude has saturated.

One might wonder how does the amplitude of this instability depend on the amplitude of the incident signal. Our results indicate that close to the finger tip, the larger the amplitude of the incident signal, the larger the amplitude of the instability. This can be seen in Fig. 8. However, far from the finger tip, we find that the saturation value for the ampli-

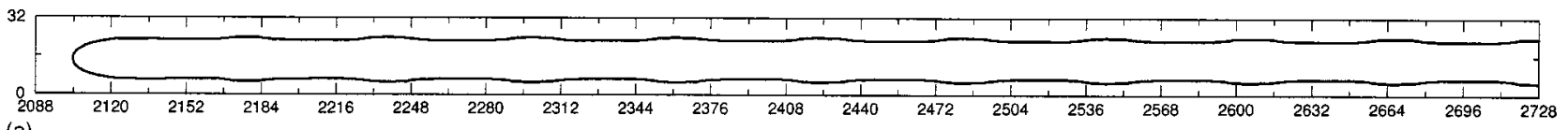

(a)

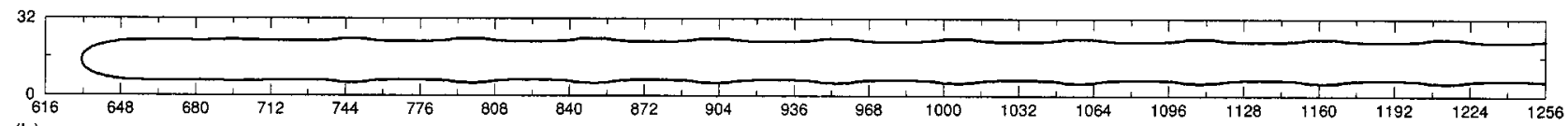

(b)

FIG. 7. The profile at the top corresponds to an incident frequency that falls in the linear region of the $\omega_{L_{2}}$ vs $\omega$ curve, whereas the profile at the bottom corresponds to a frequency that falls in the transition region of the same curve. The distance, $d_{s}$ at which the amplitude saturates is of the order of $d_{s} \approx \Lambda$ and $d_{s} \approx 2 \Lambda$, respectively. These are regimes that would be easier to observe experimentally. 


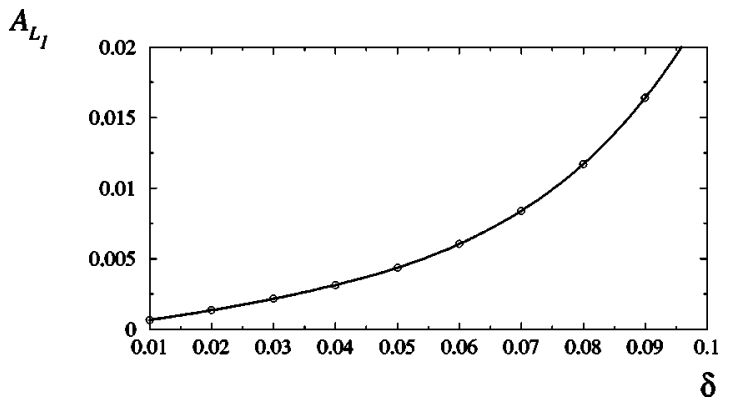

FIG. 8. Amplitude of the lateral instability measured at a fixed distance $L_{1}=40 \Delta x$ as a function of the amplitude of the incident signal.

tude of the instability is independent of the amplitude of the incident signal. Moreover, the smaller the amplitude of the incident signal, the larger the distance from the tip at which the amplitude saturates, and, therefore, the harder it becomes to observe it experimentally.

\section{B. Temporal noise}

Results of the previous section indicate that the system selects a frequency for a wide range of incident frequencies. For linear equations, this would indicate that the system acts as a selective noise amplifier. Nevertheless, our phase-field equations contain the full nonlinear behavior of the hydrodynamic equations. We therefore decided to study the effect of a dynamic signal consisting of white noise, which contains a combination of several modes.

We perturb the system by adding a random signal to a constant pressure gradient at each time step. In our model, this is implemented by choosing $g(t)$ in Eq. (7) to be a random number between -1 and 1 at each time step. The value of $\delta$ in Eq. (7) is chosen to be $\delta=0.05$.

Just as in the oscillatory case, the finger develops a lateral instability, which is born close to the finger tip, and propagates toward the sides of the finger. The amplitude of the lateral instability grows as the perturbation propagates away

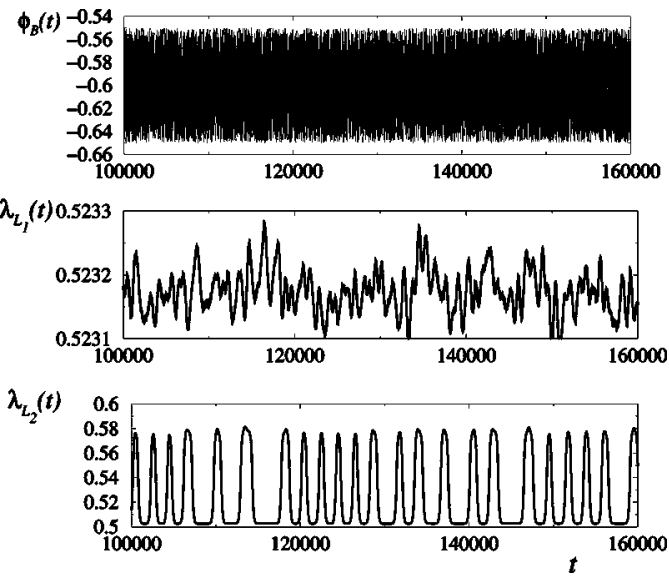

FIG. 9. Time dependence for the bulk value of the order parameter and of the finger width at distances $L_{1}$ and $L_{2}$ for the temporal noise case. For the simulation, $\delta=0.05$.

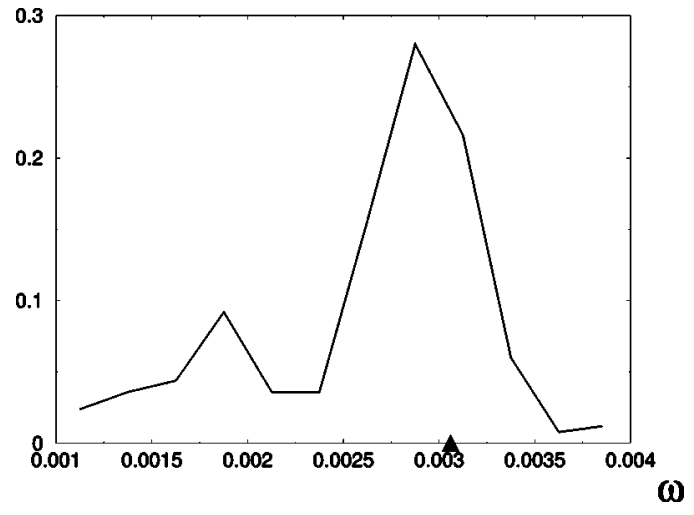

FIG. 10. Normalized distribution of response frequencies for an incident signal consisting of temporal noise. For the simulation, $\delta$ $=0.05$. For reference, the characteristic frequency of the flow $\omega_{\infty}$ is indicated in the coordinate axis with a triangle.

from the tip, and it reaches an almost constant value. From the initial random perturbation, some modes grow and some modes decay as the perturbation propagates far from the tip. In Fig. 9, we show the driving signal consisting of white noise overimposed to a constant value of the bulk order parameter. We then show the oscillations of the finger width close to the tip in which some of the modes have already decayed. Finally, we see the oscillations of the finger width far from the tip in which some of the modes have grown and the amplitude of the oscillations has reached an almost constant value. The final state has a distribution of frequencies with a peak at a value close to the characteristic frequency of the flow at infinity, that is, close to the value of the frequency selected in the oscillatory case. This can be seen in Fig. 10. This indicates that, despite the nonperiodicity of the lateral instability, it undergoes a process of mode selection. Figure 3(b) shows the nonperiodic lateral instability for a finger with temporal noise.

\section{DISCUSSION}

In the laboratory frame of reference, once the amplitude of the instability saturates, the shape remains stationary. From the tip frame of reference, the instability propagates far from the tip with a velocity equal to the average finger velocity $U$. Therefore, the expected wavelength of the instability is given by

$$
\Lambda=\frac{U}{\nu_{\text {selected }}} .
$$

The frequency selected for this long-wavelength lateral instability is roughly the characteristic frequency of the system at infinity; that is, $\nu_{\infty}=V_{\infty} / W$, therefore,

$$
\Lambda \approx \frac{U}{\nu_{\infty}}=\frac{W}{\lambda} .
$$

For fast normal fingers the finger width is close to half of the channel width, so $\lambda \approx 0.5$. This means that the expected wavelength for the lateral instability is close to twice the cell width, $\Lambda \approx 2 W$. The instability that we are reporting is a 
long-wavelength instability. This fact by itself implies the need for long channels, but there is another element to be considered. From simulations, we observe that for fingers driven by an oscillatory signal in the selection zone, with an incident amplitude of $\delta=0.09$, the distance $d_{s}$ at which the amplitude saturates is $d_{s} \approx 10 \mathrm{~W}$. This means that, not only will the wavelength of the instability be large, but it will take long for the instability to appear. For example, in an experiment with a cell $10 \mathrm{~cm}$ wide, the distance from the finger tip in which the lateral instability would be observable would be of the order of $1 \mathrm{~m}$ and the wavelength of the lateral instability of the order of $20 \mathrm{~cm}$. This fact becomes worse for the studied case of temporal noise because, in this case, the amplitude of the incident signal used was $\delta=0.05$ [see Fig. 3(b)]. In this case the distance $d_{s}$ at which the amplitude saturates is $d_{s} \approx 20 W$, which in our previous example of cell dimensions, would imply that the distance from the finger tip in which the lateral instability would be observable would be of the order of $2 \mathrm{~m}$. This is why this lateral instability has not been observed for most of experimental situations reported in literature, even when natural noise is always present on the system.

There is one experiment reference that does report lateral fluctuations in normal Saffman fingers [20]. The cells in this case were very wide and very long. We believe that such lateral fluctuations are related to the instability reported here, thus, we make a comparison to the extent possible. For large aspect ratios $W / b$ fluctutations are more easily visible for all flow rates, so we compare our computed expected wavelength for the instability to the experiment results of this case. Take for example the finger in Fig. 1(c) of Ref. [20]. For this finger, a channel of width $W \approx 20 \mathrm{~cm}$ was used and the fraction of the channel occupied by the finger was roughly $\lambda \approx 0.5$. According to our calculations, this should give an expected wavelength of the order of $\Lambda \approx 40 \mathrm{~cm}$, which is roughly what can be visually measured from the experimental figure. The finger, however, is not long enough to allow for comparison to our frequency distribution because, despite having the longest channels reported in literature, the wavelength of the instability is also very long, therefore, there are only 5 or 6 appreciable maxima.

Another result of our numerical integration that agrees with the experiment results of Ref. [20] is that the larger the velocity of finger propagation, the more stable the tip is. What happens, according to our studies, is that the distance at which the instability saturates is larger for larger velocities. Therefore, close to the finger tip, the finger looks more similar to the steady-state finger. Just as mentioned in Ref. [20], our numerical integration indicates that even when the finger tip appears exactly like the classical Saffman-Taylor finger, with sufficient resolution fluctuations can still be measured close to the finger tip for all velocities. In order to illustrate this point we plot the amplitude of the oscillations close to the finger tip versus tip velocity in Fig. 11. We can see that the larger the velocity, the smaller the amplitude of the oscillations. On the other hand, far from the finger tip, our results indicate that the amplitude of the instability is independent of finger velocity. These results imply that for the experimental cases in which the finger tip appears like the classical Saffman-Taylor finger, longer channels would

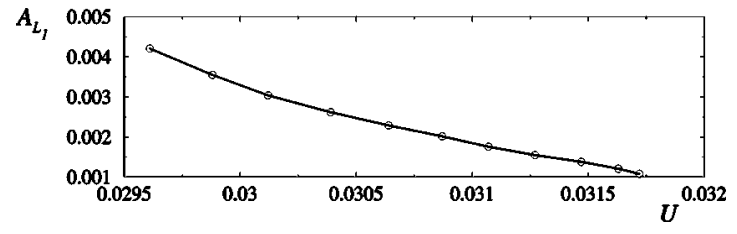

FIG. 11. Amplitude of the instability close to the finger tip as a function of the average finger-tip velocity.

be needed in order to observe fluctuations. One thing that differs between the experiments reported in Ref. [20] and our numerical integration is the distance from the tip at which fluctuations are observable, this one being larger for the numerical integration. We are currently studying the possibility of such a discrepancy being related to the presence of small spatial variations present in the cell, but this is out of the scope of the present paper.

If experiments were to be conducted for an oscillatory pressure drop, the following considerations should be taken into account. For oscillatory fingers, selection is observed for incident frequencies larger than the characteristic frequency of the finger $\nu_{\text {finger }}=U / \lambda W$. Therefore, in order to observe selection, it would be necessary to apply frequencies larger than $\nu_{\text {finger }}$. On the other hand, the linear and transition regimes of the oscillatory fingers are not as hard to observe. For instance, for the oscillatory case with $\delta=0.09$, the distance $d_{s}$ at which the amplitude saturates, in terms of the wavelength for the instability, is of the order of $d_{s} \approx \Lambda$ for the linear regime and of the order of $d_{s} \approx 2 \Lambda$ for the transition zone. This can be seen in Fig. 7. Therefore, in order to observe the linear regime of the lateral instability, it would be enough to apply an incident frequency close to the frequency characteristic of infinity. The transition zone should be observed for incident frequencies between the two characteristic frequencies of the system, that is, between $\nu_{\infty}$ and $\nu_{\text {finger }}$.

\section{CONCLUSIONS}

An oscillatory signal overimposed on a constant pressure gradient produces a low-amplitude, long-wavelength lateral instability on normal Saffman-Taylor fingers. The instability undergoes a mode selection process and reaches a final state with a shape of a single finger whose sides have lowamplitude strictly periodic undulations. The selected frequency corresponds roughly to the characteristic frequency of the system at infinity. This implies that the selected wavelength of the lateral instability for fast fingers will be close to twice the channel width. Also, the distance from the tip for the lateral instability to appear is several times the expected wavelength. So in order to observe this, very long channels are necessary. For low frequencies, there is a linear regime in which the response frequency is the same as the incident frequency and a transition zone for which the response frequency is half of the incident frequency. For these cases, it should be easier to observe the instability because the distance from the tip for the instability to be observable is of the 
order of once or twice the expected wavelength. When temporal noise is added to the system, the same lateral instability is observed. The distribution of frequencies has a peak at a frequency close to the frequency selected in the oscillatory case. We believe that our work could help us to understand the lateral fluctuations along the finger sides reported in Ref. [20] and we have made some qualitative comparisons between our numerical integration in the presence of temporal noise and experimental results. However, we are currently exploring the relation of such lateral fluctuations with small spatial variations present in the cell. We believe that in order to understand results from Ref. [20], further studies are needed.

\section{ACKNOWLEDGMENTS}

This work was partially supported by DGAPA through Grant No. PAPIIT IN117802-2 and by CONACYT through project 38538. A.H.M. acknowledges financial support of the Dirección General de Investigación under project No. BFM2003-07749-C05-04. The authors thank Ana Lacasta and Antonio del Río for very useful discussions.
[1] Branching in Nature, edited by V. Fleury, J.-F. Gouyet, and M. Léonetti (Springer-Verlag, Berlin, 2001).

[2] E. Ben-Jacob and H. Levine, Adv. Phys. 49, 395 (2000).

[3] J. Gollub and J. Langer, Rev. Mod. Phys. 71, S396 (1999).

[4] Solids Far from Equilibrium, edited by C. Godrèche (Cambridge University Press, Cambridge, England, 1992).

[5] A. Dougherty, P. Kaplan, and J. Gollub, Phys. Rev. Lett. 58, 1652 (1987).

[6] A. Dougherty and J. Gollub, Phys. Rev. A 38, 3043 (1988).

[7] M. Barber, A. Barbieri, and J. Langer, Phys. Rev. A 36, 3340 (1987).

[8] R. Pieters, Phys. Rev. A 37, 3126 (1988).

[9] R. Pieters and J. Langer, Phys. Rev. Lett. 56, 1948 (1986).

[10] Y. Couder, N. Gérard, and M. Rabaud, Phys. Rev. A 34, 5175 (1986).

[11] M. Rabaud, Y. Couder, and N. Gerard, Phys. Rev. A 37, 935 (1988).

[12] P. Bouissou, A. Chiffaudel, B. Perrin, and P. Tabeling, Europhys. Lett. 13, 89 (1990).

[13] X. Qian and H. Cummins, Phys. Rev. Lett. 64, 3038 (1990).
[14] Perspectives in Fluid Dynamics, edited by G. K. Batchelor, H. K. Moffat, and M. G. Worster (Cambridge University Press, Cambridge, England, 2000).

[15] E. Ben-Jacob, R. Godbey, N. Goldenfeld, J. Koplik, H. Levine, T. Mueller, and L. Sander, Phys. Rev. Lett. 55, 1315 (1985).

[16] E. Decker, J. Ignés-Mullol, A. Baratt, and J. Maher, Phys. Rev. E 60, 1767 (1999).

[17] K. McCloud and J. Maher, Phys. Rep. 260, 139 (1995).

[18] S. Sarkar and D. Jasnow, Phys. Rev. A 39, 5299 (1989).

[19] D. Bensimon, Phys. Rev. A 33, 1302 (1986).

[20] M. Moore, A. Juel, J. Burgess, W. McCormick, and H. Swinney, Phys. Rev. E 65, 030601 (2002).

[21] P. Pelcé, Dynamics of Curved Fronts (Academic, San Diego, 1988).

[22] R. Folch, J. Casademunt, A. Hernández-Machado, and J. Ramírez-Piscina, Phys. Rev. E 60, 1724 (1999).

[23] R. Folch, J. Casademunt, A. Hernández-Machado, and J. Ramírez-Piscina, Phys. Rev. E 60, 1734 (1999).

[24] A. Hernández-Machado, A. M. Lacasta, E. Mayoral, and E. Corvera-Poiré, Phys. Rev. E 68, 046310 (2003). 